\title{
An Organic D- $\pi$-A Dye for Record Efficiency Solid-State Sensitized Heterojunction Solar Cells
}

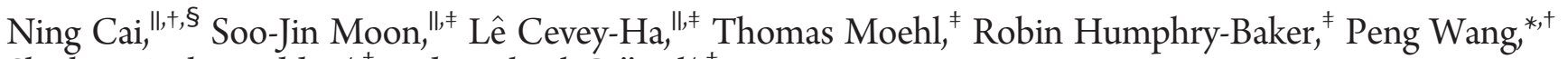 \\ Shaik M. Zakeeruddin,, ${ }^{*, \neq}$ and Michael Grätzel ${ }^{*, \neq}$ \\ ${ }^{\dagger}$ State Key Laboratory of Polymer Physics and Chemistry, Changchun Institute of Applied Chemistry, Chinese Academy of Sciences, \\ Changchun 130022, China \\ ${ }^{\ddagger}$ Laboratory of Photonics and Interfaces, Institute of Chemical Sciences and Engineering, Ecole Polytechnique Fédérale de Lausanne \\ (EPFL), Station 6, CH-1050 Lausanne, Switzerland \\ ${ }^{\S}$ Graduate School, Chinese Academy of Sciences, Beijing 100039, China
}

Supporting Information

ABSTRACT: The high molar absorption coefficient organic $D-\pi$-A dye C220 exhibits more than $6 \%$ certified electric power conversion efficiency at AM 1.5G solar irradiation (100 $\mathrm{mW} \mathrm{cm}^{-2}$ ) in a solid-state dye-sensitized solar cell using $2,2^{\prime}, 7,7^{\prime}$-tetrakis ( $N, N$-dimethoxyphenylamine)-9, $9^{\prime}$-spirobifluorene (spiro-MeOTAD) as the organic hole-transporting material. This contributes to a new record (6.08\% by NREL) for this type of sensitized heterojunction photovoltaic device. Efficient charge generation is proved by incident photon-tocurrent conversion efficiency spectra. Transient photovoltage and photocurrent decay measurements showed that the enhanced performance achieved with C220 partially stems from the high charge collection efficiency over a wide potential range.

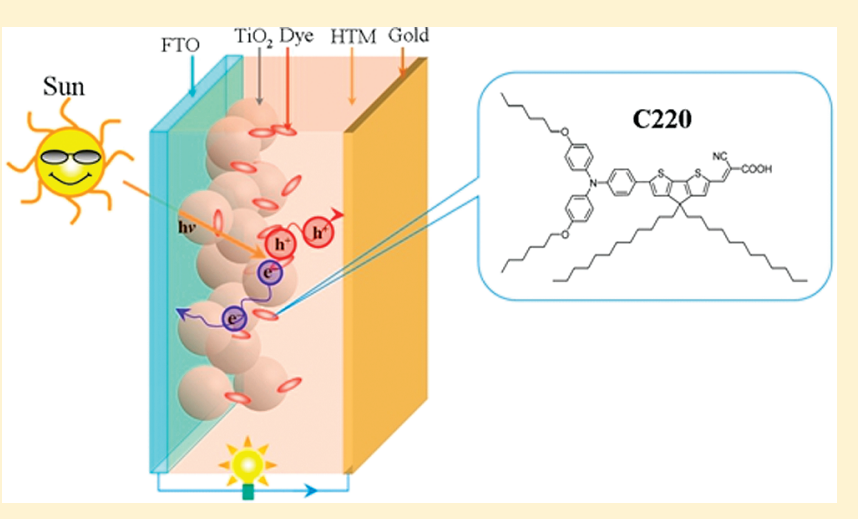

KEYWORDS: Solid-state dye-sensitized solar cells, D- $\pi$-A dye, electron injection, charge collection

$\mathrm{D}^{\prime}$ ye-sensitized solar cells (DSCs) have attracted significant attention as low-cost alternatives to conventional semiconductor photovoltaic devices. ${ }^{1,2}$ These cells are composed of a nanocrystalline wide band gap semiconductor oxide, e.g., $\mathrm{TiO}_{2}$ deposited on a transparent conducting oxide (TCO) glass substrate. A molecular sensitizer is linked via an anchoring group to the oxide surface. Upon light excitation, it injects an electron into the conduction band of the semiconductor. Ruthenium complexes as molecular sensitizers have shown impressive solarto-electric power conversion efficiencies (PCE) in liquid electrolyte based devices, with the PCE reaching over $11 \%$ under standard AM1.5G full sunlight. ${ }^{3-7}$ Recently, metal-free organic dyes have attracted increasing attention due to their high structural flexibility, high molar extinction coefficient, low toxicity, and environmental friendliness. DSCs employing organic dyes featuring an electron donor and acceptor moiety connected by a $\pi$-conjugation bridge have reached $\sim 10 \%$ efficiency with liquid electrolytes. ${ }^{8-12}$ They can be easily modified to tune their properties for device performance optimization. One of the main factors limiting the performance of DSCs with organic dyes is the formation of dye aggregates on the surface of the semiconductor oxide, leading to quenching of the excited state of the dye molecules and lowering the overall performance of the device. ${ }^{13}$ Appropriate molecular structural modifications have been conceived to avoid this undesirable aggregation process. ${ }^{14-16}$
In solid-state DSCs (ssDSCs) a solid hole-transporting material (HTM) is employed to replace the liquid redox electrolyte which is responsible for the dye regeneration and hole transfer to the counter electrode. Typically, solid HTMs have shorter charge carrier diffusion lengths than liquid redox electrolytes. Due to incomplete pore filling of thicker $\mathrm{TiO}_{2}$ films with solid HTMs, the ssDSCs are restricted to using thinner titania films. High molar extinction coefficient dyes are of great importance for this type of device in order to enable the use of thinner films. On the basis of this strategy, we have designed and synthesized the 4 , $4^{\prime}$-didodecyl-4H-cyclopenta[2,1-b:3,4- $\left.b^{\prime}\right]$ dithiophene (CPDT) segment which is used as the spacer between the donor and the acceptor group of a metal-free organic sensitizer (called C220). Here, we report on the photovoltaic performance of the $\mathbf{C 2 2 0}$ sensitizer in a ssDSC using spiro-MeOTAD as the organic holetransporting material.

The molecular structure of the C220 dye is presented in Figure 1, which is analogous to the recently reported C218 dye except that dodecyl substituents replace the two hexyl substituents on the CPDT segment. ${ }^{17}$ The detailed synthesis of C220 is described in the Supporting Information. The electronic

Received: November 18, 2010

Revised: February 7, 2011

Published: March 04, 2011 


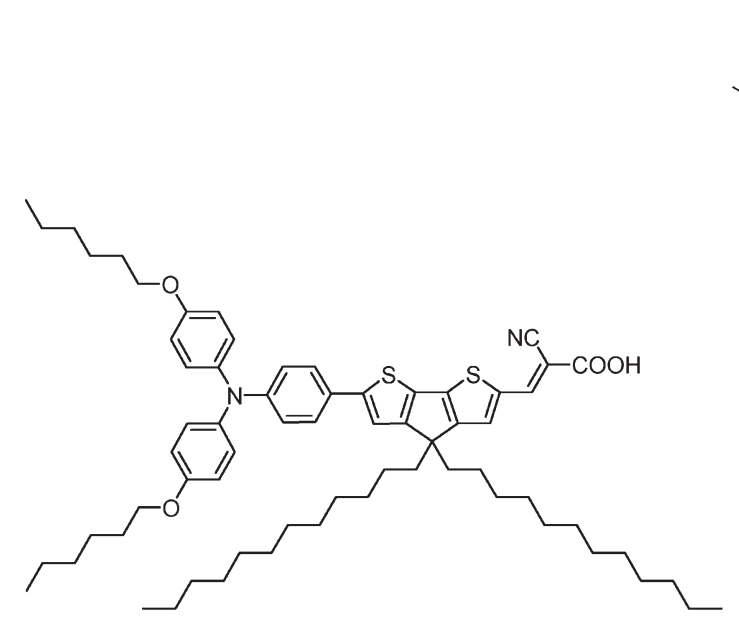

(a)

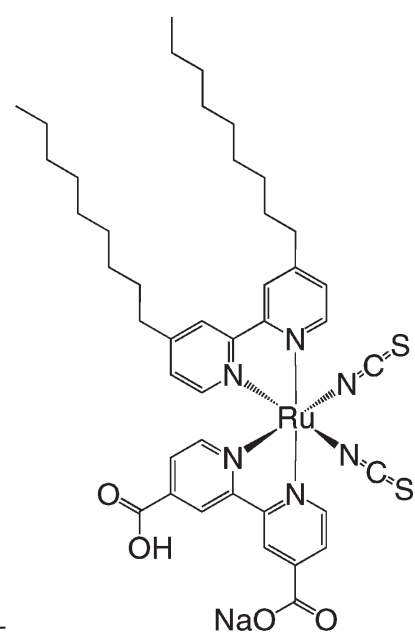

(b)

Figure 1. The molecular structures of $\mathbf{C 2 2 0}$ (a) and Z907 (b).

absorption spectrum of the $\mathbf{C 2 2 0}$ dye in chloroform exhibits a maximum molar absorption coefficient $\varepsilon$ of $5.5 \times 10^{4} \mathrm{M}^{-1} \mathrm{~cm}^{-1}$ at $555 \mathrm{~nm}$, which is nearly five times higher than that of the standard Z907 ruthenium sensitizer $\left(\varepsilon=1.2 \times 10^{4} \mathrm{M}^{-1} \mathrm{~cm}^{-1}\right.$ at $521 \mathrm{~nm}$ ). The redox potential of $\mathbf{C 2 2 0}$ in DMF was measured by employing the ultramicroelectrode technique in conjunction with square-wave voltammetry. The energy offset of the LUMO $(-3.42 \mathrm{eV})$ of the dye molecule with respect to the titania conduction band edge $(\mathrm{ca} .-4.0 \mathrm{eV})^{18}$ provides the thermodynamic driving force for charge injection. The energy offset of the HOMO $(-5.01 \mathrm{eV})$ relative to that of the spiro-MeOTAD $(-4.88 \mathrm{eV})^{19}$ presents enough driving force for the dye regeneration process.

Figure 2a shows the certified $J-V$ characteristics of a ssDSC using the C220 dye as the sensitizer, spiro-MeOTAD as the HTM, and a $2.0 \mu \mathrm{m}$ thick transparent titania film measured at the National Renewable Energy Laboratory (USA) under standard global AM1.5 conditions. The short-circuit photocurrent $\left(J_{\text {sc }}\right)$ is $9.74 \mathrm{~mA} \mathrm{~cm}^{-2}$, the open-circuit photovoltage $\left(V_{\mathrm{oc}}\right) 881 \mathrm{mV}$ and the fill factor (FF) 0.71 , yielding a PCE of $6.08 \%$. This constitutes a new record for this type of sensitized heterojunction device, compared to the highest validated conversion efficiency of 5\% reported using a ruthenium sensitizer. ${ }^{20}$ To the best of our knowledge this is the first time that a certified efficiency of over $6 \%$ has been reached with a sensitized solid-state heterojunction solar cell. The PCE of a champion cell measured under AM1.5G standard conditions in our laboratory reached even 6.5\% (with $V_{\text {oc }}=860 \mathrm{mV}, J_{\mathrm{sc}}=10.90 \mathrm{mAcm}^{-2}$, and $\left.\mathrm{FF}=0.69\right)$. Under similar conditions $\left(2.2 \mu \mathrm{m}\right.$ of $\mathrm{TiO}_{2}$ film $)$ a ssDSC employing the Z907 dye gives a PCE of $3.2 \%\left(J_{\mathrm{sc}}=7.35 \mathrm{~mA} \mathrm{~cm}^{-2}, V_{\mathrm{oc}}=790\right.$ $\mathrm{mV}$, and $\mathrm{FF}=0.56$ ).

The incident photon-to-current conversion efficiency (IPCE) spectra for the two sensitizers are shown in Figure 2b. For C220 the IPCE is close to $70 \%$ from $450 \mathrm{~nm}$ up to $570 \mathrm{~nm}$. For Z907 we observe that a lower maximum IPCE (slightly higher than $50 \%)$ with a narrower plateau was obtained. This difference in IPCE shows that the enhanced light absorption capabilities of the C220 dye leads to enhanced charge carrier injection which is confirmed by the $J-V$ characteristic. Integration of the IPCE spectra over the AM1.5G standard solar emission spectrum leads to the projected $J_{\text {sc }}$ values for the $\mathbf{C 2 2 0}$ and Z907 dyes of 9.3 and
$7.0 \mathrm{~mA} \mathrm{~cm}^{-2}$, respectively, which is in good agreement with the measured values.

The electron injection efficiency $\left(\eta_{\text {inj }}=1-\tau_{\mathrm{ZrO}_{2}} / \tau_{\mathrm{TiO}_{2}}\right)$ can be estimated from the photoluminescence measurements, since the emission process is usually considered to be in direct competition with the electron injection process unless the emission arises from aggregates or from dye molecules that are loosely bound to the titania surface. As shown in Figure S1 (Supporting Information), injection yields can be estimated by the integral areas under the emission decay traces or from the half-life of the emission decays reported by Durrant et al. ${ }^{21}$ (see Table S1, Supporting Information). Both methods showed higher injection yields for the C220 (0.97 and 0.92) in comparison with those of Z907 (0.88 and $0.85)$. A third way to determine the $\eta_{\text {inj }}$ by using wavelengthdependent emission could only be applied to the C220 due to limitations of the detector of the experimental setup in the infrared region, giving a value of 0.97 . Since the time resolution of the laser system technique used to determine the emission lifetimes is in the picosecond domain, the reported injection efficiency for the sensitizers is only a lower bound as the emission may arise from dye molecules that are only loosely associated with the titania surface or are present as aggregates.

Note that apart from the higher photocurrents C220 also yields around $70 \mathrm{mV}$ larger $V_{\text {oc }}$ in a ssDSC that of $\mathrm{Z} 907$ dye. A change in $V_{\text {oc }}$ can result from a lower Fermi level in the HTM (increased concentration of holes) or an upward shift of the conduction band edge of the $\mathrm{TiO}_{2}$ (by changing the dipoles at the $\mathrm{TiO}_{2}$ surface). Since similar experimental conditions were applied in the fabrication of ssDSCs with the two different dyes, the HTM Fermi level is unlikely to shift noticeably. Thereby, higher open-circuit photovoltage achieved with C220 compared to that of Z907 probably stems from an upward shift of the titania conduction band edge.

With these high-performance ssDSC devices, transient photocurrent and photovoltage measurements were conducted to investigate the internal electrical parameters. ${ }^{22,23}$ This technique enables the determination of the effective electron transport time ( $\left.\tau_{\text {trans }}\right)$, the electron lifetime $\left(\tau_{\mathrm{e}}\right)$, and the charge collection efficiency $\left(\eta_{\mathrm{cc}}\right)$.

In transient measurements, the photovoltage decay can only be ascribed to the interfacial charge recombination and thus gives 
(a)

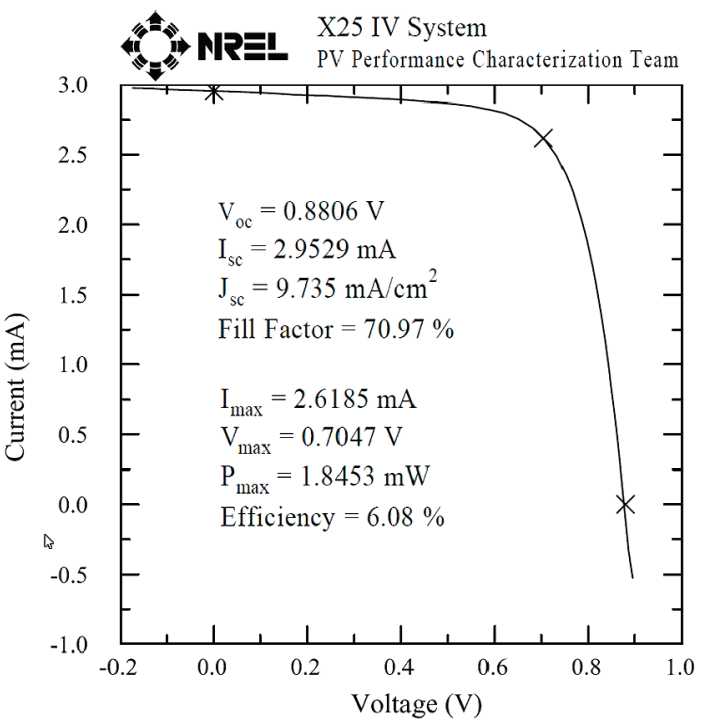

(b)

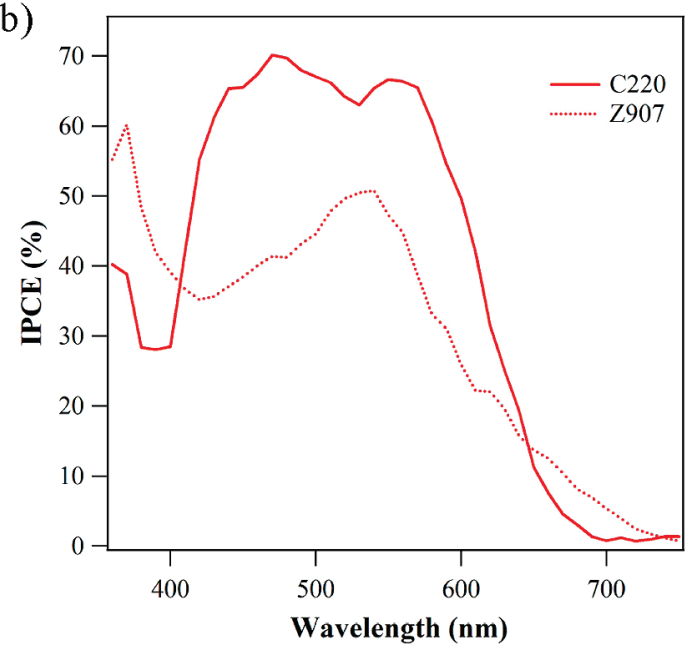

Figure 2. (a) $J-V$ characteristics of a ssDSC sensitized with C220 measured by the NREL photovoltaic calibration laboratory under standard reporting conditions, i.e., illumination with AM1.5G sunlight (intensity $100 \mathrm{~mW} \mathrm{~cm}^{-2}$ ) and at $298 \mathrm{~K}$. Cell active area tested (with a mask): $0.3033 \mathrm{~cm}^{2}$. (b) Incident photon-to-current conversion efficiency (IPCE) spectra of $\mathbf{C 2 2 0}$ and Z907 dye-sensitized devices.

direct access to the electron lifetime $\tau_{\text {e, }}$ while the lifetime $\left(\tau_{\text {signal }}\right)$ of the photocurrent decay is collectively contributed by $\tau_{\text {trans }}$ and $\tau_{\mathrm{e}}$ of the electrons. Thereby, transport time $\left(\tau_{\text {trans }}\right)$ could be calculated with the equation: $\left(1 / \tau_{\text {signal }}=1 / \tau_{\text {trans }}-1 / \tau_{\mathrm{e}}\right)$.

In Figure 3 the results of the transient measurements at 1 sun are presented. For the ssDSC device with C220 we observe a clearly shorter transport time compared to that of Z907 (at short circuit $\left.\tau_{\text {trans,C220 }} / \tau_{\text {trans,Z907 }}=0.66\right)$ whereas the lifetime of electrons is only slightly lower for the C220 device at short circuit condition. In general it can be seen that for the Z907sensitized ssDSC the $\tau_{\text {trans }}$ increases faster and $\tau_{\mathrm{e}}$ drops faster with increasing forward bias than when $\mathbf{C 2 2 0}$ is used as sensitizer, explaining the lower open circuit voltage and lower fill factor obtained with the former device. Especially the electron lifetime for $\mathrm{Z907}$ starts to drop at lower forward bias $(\sim 0.2 \mathrm{~V})$ as compared to $\mathbf{C 2 2 0}(\sim 0.4 \mathrm{~V})$.

The same tendency can be observed when the charge collection efficiency, $\eta_{\mathrm{cc}}=1 /\left(1+\left(\tau_{\text {trans }} / \tau_{\mathrm{e}}\right)\right)$, ${ }^{24}$ is plotted against

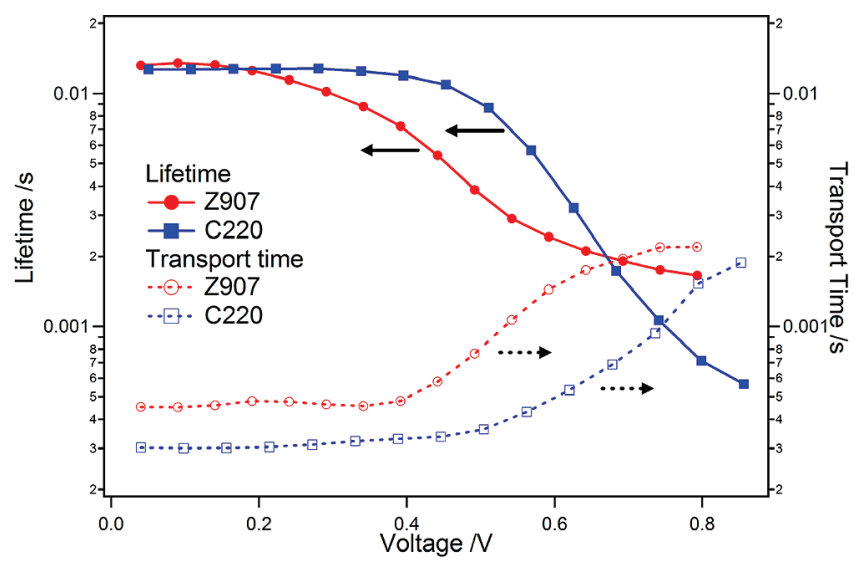

Figure 3. Electron lifetime (solid squares and circles) and transport time (open squares and circles) determined by photocurrent and photovoltage decay measurements of a ssDSC fabricated with C220 (blue) and Z907 (red). Measurements were performed at 1 sun illumination.

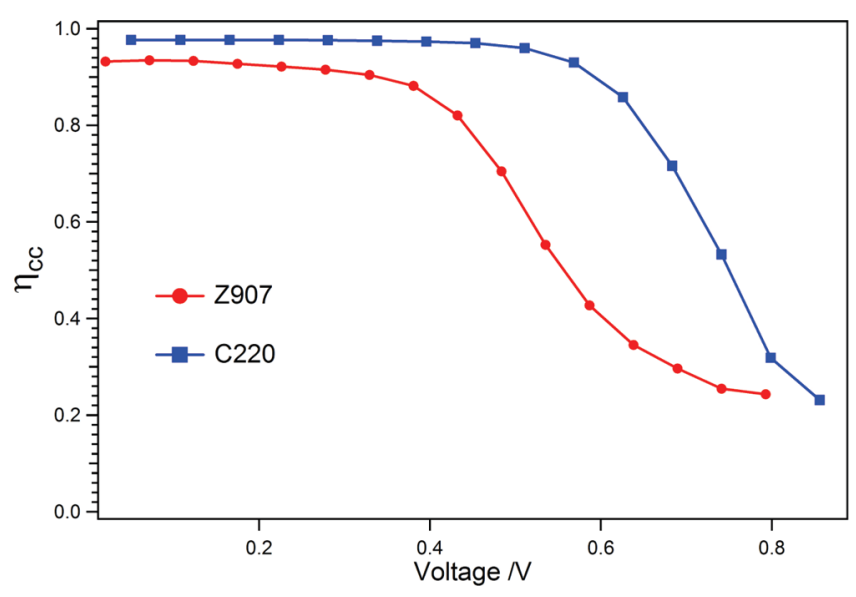

Figure 4. Charge collection efficiency of a ssDSC sensitized with C220 (blue) and Z907 (red) at 1 sun illumination.

applied potential. As can be observed in Figure 4, the $\eta_{\mathrm{cc}}$ derived from the transient photocurrent and photovoltage decay measurement is over $90 \%$ for both dyes at potential bias below $0.3 \mathrm{~V}$. The charge collection efficiency is slightly higher near short circuit conditions for the ssDSC with C220. When the forward bias is increased, the collection efficiency of devices sensitized by Z907 drops faster than with C220. The fact that the collection efficiency of DSCs with C220 stay stable over a wider potential range is one of the main reasons for the better performance of these DSCs. In addition the light harvesting and injection contribute to the overall higher efficiency. The high $V_{\mathrm{oc}}$ and the high injection of charge carriers paired with the good charge collection efficiency leads to an impressive overall efficiency of more than $6 \%$ under 1 sun illumination of the ssDSC.

In summary, we can see the specific chemical design of this novel D- $\pi$-A dye leads to higher photovoltaic performance. We could demonstrate that the $\mathbf{C 2 2 0}$ dye has a higher solar light harvesting capacity compared to $\mathrm{Z907}$ and a near unity quantum yield for electron injection. The experiments confirm that the higher device performance is due to the higher charge generation efficiency of the $\mathbf{C 2 2 0}$ and higher collection efficiency relative to Z907 over a wide potential range. This work shows the importance of designing new dyes with high molar extinction 
coefficient to further decrease the film thickness of ssDSC devices leading to higher charge carrier concentrations under illumination. It paves the way for future structural design of new metal-free sensitizers to use in thin film devices.

Experimental Part and Device Fabrication. 1. Device Fabrication. For the solid-state devices, a compact $\mathrm{TiO}_{2}$ layer was first deposited onto the FTO substrate by spray pyrolysis, onto which $23 \mathrm{~nm}$ sized $\mathrm{TiO}_{2}$ particles were deposited by screen printing to obtain around $2.0 \mu \mathrm{m}$ thick mesoporous film. After sintering the $\mathrm{TiO}_{2}$ layer at $500{ }^{\circ} \mathrm{C}$, the film was cooled to room temperature and immersed for $6 \mathrm{~h}$ in $0.02 \mathrm{M}$ aqueous $\mathrm{TiCl}_{4}$. The film was then rinsed with deionized water, annealed in air at $450{ }^{\circ} \mathrm{C}$ for $20 \mathrm{~min}$, and cooled to $50^{\circ} \mathrm{C}$ before immersing it in the above-mentioned dye solution for sensitization $(0.1 \mathrm{mM}$ in tertbutanol and acetonitrile at a volume ratio of 1:1). The HTM solution containing 0.17 M Spiro-MeOTAD (Merck), $0.11 \mathrm{mM}$ tert-butylpyridine, and $0.21 \mathrm{mM} \mathrm{LiN}\left(\mathrm{CF}_{3} \mathrm{SO}_{2}\right)_{2}$ in chlorobenzene was used. We deposited this solution onto the dye-coated $\mathrm{TiO}_{2}$ film, leaving it to penetrate into the pores of the $\mathrm{TiO}_{2}$ layer for $45 \mathrm{sec}$ prior to spin coating. Finally, a $200 \mathrm{~nm}$ of Ag contact was deposited onto the organic semiconductor to close the cell.

2. Electrochemical Characterization. A CHI660C electrochemical workstation was used for square-wave voltammetric measurements in combination with a three-electrode electrochemical minicell equipped with a $5 \mu \mathrm{m}$ radius $\mathrm{Pt}$ ultramicroelectrode as the working electrode. A Pt wire and a silver wire were used as the counter and quasi-reference electrodes, respectively. The redox potentials were calibrated with ferrocene as the internal reference. The HOMO and LUMO values were transformed via the equation $E_{\text {LUMO/HOMO }}=-e\left(4.88+V_{\text {redox }}\right),{ }^{12}$ where $V_{\text {redox }}$ is the onset potential versus ferrocene of reduction or oxidation of $\mathbf{C 2 2 0}$.

3. Optical Characterization. Steady-state emission and emission dynamics were measured on a Horiba Jobin-Yvon Fluorolog322. The same optical detection channel was used to collect the light at right angles with respect to the excitation channel for both the steady-state signal and the dynamic signal. $\mathrm{TiO}_{2}$ and $\mathrm{ZrO}_{2}$ films, 3-4 $\mu \mathrm{m}$ thick, were immersed in C220, $0.1 \mathrm{mM}$ solution in tert-butanol, and acetonitrile (1:1 volume ratio), for 1 $\mathrm{h}$ and afterward rinsed with acetonitrile. The emission spectra were recorded at room temperature and ambient atmosphere and then photometrically corrected. The emission lifetime was measured using the time correlated single photon counting (TCSPC) configuration, with a $460 \mathrm{~nm}$ laser diode having a pulse width at fwhm of about 700 ps. The effective instrument time resolution is about $50 \mathrm{ps}$ after deconvolution.

4. Transient Photocurrent and Photovoltage Decay Measurements. White light bias was generated by an array of diodes (Lumiled model LXHL-NWE8 white star) in combination with red light pulsed diodes (LXHLND98 redstar, $0.2 \mathrm{~s}$ square pulse width, $100 \mathrm{~ns}$ rise and fall time) for the perturbation excitation, controlled by a fast solid-state switch. The voltage dynamics were recorded via a Keithley 2602 sourcemeter. The voltage decay measurements were performed from zero current $\left(V_{\mathrm{oc}}\right)$ over a range of fixed current intervals on the photocurrent-voltage curve to mimic the $J-V$ characteristic of the device while measuring the transients. Small perturbation transient photocurrent measurements were performed in a similar way to the open-circuit voltage decay measurements but here holding a fixed potential while measuring the photocurrent transients. The decays were fitted with a double exponential decay. To check on the RC limitation of the measurement setup, the pulse charge rate and the voltage decay rate were compared and showed no limitation.

\section{ASSOCIATED CONTENT}

S Supporting Information. Additional information on synthesis of C220 and emission measurements. This material is available free of charge via the Internet at http://pubs.acs.org.

\section{AUTHOR INFORMATION}

\section{Corresponding Author}

*E-mail: peng.wang@ciac.jl.cn, shaik.zakeer@epfl.ch, michael. graetzel@epfl.ch.

\section{Author Contributions}

"These authors have equal contribution.

\section{ACKNOWLEDGMENT}

N.C. and P.W. thank the National Key Scientific Program (No. 2007CB936700), the National Science Foundation of China (No. 50973105), and the Hundred Talent Program of Chinese Academy of Sciences for the financial support. This publication is partially based on work supported by the Center for Advanced Molecular Photovoltaics (Award No KUS-C1-01521), made by King Abdullah University of Science and Technology (KAUST). Financial support from the Swiss National Science Foundation is also gratefully acknowledged. M.G. thanks the ECR advanced grant agreement (No. 247404) under the CEMesolight project funded by the European community's 7 th FWP. We are grateful to Mr. P. Comte for $\mathrm{TiO}_{2}$ nanoparticles preparations and Mr. Jean-David Decoppet and Dr. Paul Liska for helping in preparing and performing the NREL measurements.

\section{REFERENCES}

(1) O’Regan, B.; Grätzel, M. Nature 1991, 353, 737-740.

(2) Grätzel, M. Nature 2001, 414, 338-344.

(3) Nazeeruddin, M. K.; De Angelis, F.; Fantacci, S.; Selloni, A.; Viscardi, G.; Liska, P.; Ito, S.; Bessho, T.; Grätzel, M. J. Am. Chem. Soc. 2005, 127, 16835-16847.

(4) Chiba, Y.; Islam, A.; Watanabe, Y.; Komiya, R.; Koide, N.; Han, L. Y. Jpn. J. Appl. Phys. 2006, 45, L638-L640.

(5) Gao, F.; Wang, Y.; Shi, D.; Zhang, J.; Wang, M. K.; Jing, X. Y.; Humphry-Baker, R.; Wang, P.; Zakeeruddin, S. M.; Grätzel, M. J. Am. Chem. Soc. 2008, 130, 10720-10728.

(6) Cao, Y. M.; Bai, Y.; Yu, Q. J.; Cheng, Y. M.; Liu, S.; Shi, D.; Gao, F.; Wang, P. J. Phys. Chem. C 2009, 113, 6290-6297.

(7) Chen, C.-Y.; Wang, M. K.; Li, J.-Y.; Pootrakulchote, N.; Alibabaei, L.; Ngoc-le, C. H.; Decoppet, J. D.; Tsai, J. H.; Grätzel, C.; Wu, C. G.; Zakeeruddin, S. M.; Grätzel, M. ACS Nano 2009, 3, 3103-3109.

(8) Hara, K.; Sato, T.; Katoh, R.; Furube, A.; Ohga, Y.; Shinpo, A.; Suga, S.; Sayama, K.; Sugihara, H.; Arakawa, H. J. Phys. Chem. B 2003, 107, 597-606.

(9) Horiuchi, T.; Miura, H.; Sumioka, K.; Uchida, S. J. Am. Chem. Soc. 2004, 126 (39), 12218-12219.

(10) Kim, S.; Lee, J. K.; Kang, S. O.; Ko, J.; Yum, J.-H.; Fantacci, S.; De Angelis, F.; Di Censo, D.; Nazeeruddin, M. K.; Grätzel, M. J. Am. Chem. Soc. 2006, 128, 16701-16707.

(11) Hagberg, D. P.; Edvinsson, T.; Marinado, T.; Boschloo, G.; Hagfeldt, A.; Sun, L. C. Chem. Commun. 2006, 2245-2247.

(12) Zeng, W. D.; Cao, Y. M.; Bai, Y.; Wang, Y. H.; Shi, Y. S.; Zhang, M.; Wang, F. F.; Pan, C. Y.; Wang, P. Chem. Mater. 2010, 22, 1915-1925.

(13) Liu, D.; Fessenden, R. W.; Hug, G. L.; Kamat, P. V. J. Phys. Chem. B 1997, 101, 2583-2590.

(14) Burfeindt, B.; Hannappel, T.; Storck, W.; Willig, F. J. Phys. Chem. 1996, 100, 16463-16465. 
(15) Sayama, K.; Tsukagoshi, S.; Hara, K.; Ohga, Y.; Shinpou, A.; Abe, Y.; Suga, S.; Arakawa, H. J. Phys. Chem. B 2002, 106, 1363-1371.

(16) Wang, Z.-S.; Hara, K.; Dan-oh, Y.; Kasada, C.; Shinpo, A.; Suga, S.; Arakawa, H.; Sugihara, H. J. Phys. Chem. B 2005, 109, 3907-3914.

(17) Li, R. Z.; Liu, J. Y.; Cai, N.; Zhang, M.; Wang, P. J. Phys. Chem. B 2010, 114, 4461-4464.

(18) De Angelis, F.; Fantacci, S.; Selloni, A. Nanotechnology 2008, 19, 424002-424008.

(19) Garcia-Canadas, J.; Fabregat-Santiago, F.; Bolink, H. J.; Palomares, E.; Garcia-Belmonte, G.; Bisquert, J. Synth. Met. 2006, 156, 944-948.

(20) Wang, M. K.; Liu, J. Y.; Cevey-Ha, N. L.; Moon, S. J.; Liska, P.; Humphry-Baker, R.; Moser, J. E.; Grätzel, C.; Wang, P.; Zakeeruddin, S. M.; Grätzel, M. Nano Today 2010, 5, 169-174.

(21) Koops, S. E.; Barnes, P. R. F.; O’Regan, B. C.; Durrant, J. R. J. Phys. Chem. C 2010, 114, 8054-8061.

(22) O’Regan, B. C.; Lenzmann, F. J. Phys. Chem. B 2004, $108,4342-4350$.

(23) Snaith, H. J.; Humphry-Baker, R.; Chen, P.; Cesar, I.; Zakeeruddin, S. M.; Grätzel, M. Nanotechnology 2008, 19, 424003-424014.

(24) Wang, Q.; Zhang, Z.; Zakeeruddin, S. M.; Grätzel, M. J. Phys. Chem. C 2008, 112, 7084-7092. 